\title{
Editorial of special section on enabling technologies for industrial and smart sensor internet of things systems
}

\author{
Cho Jaeik ${ }^{1}$ - Naveen Chilamkurti ${ }^{2}$ S. J. Wang ${ }^{3}$
}

(c) Springer Science+Business Media, LLC, part of Springer Nature 2018

Internet of things (IoT) makes human life much easier by enabling smart machines sensing, data-gathering, processing, interacting and communicating with each other in every aspect of our lives. Recently, there are growing interests in using IoT technologies in various industries. IoT is expected to provide promising solutions to many existing industrial processes and systems such as manufacturing, logistics, facility diagnostics, product inspection and power systems. Such expectations lead to rapid expansion to the new concept of Industrial IoT (IIoT) based on Smart Sensors (e.g., Industry 4.0, Smart Factory, etc.). In near future, IIoT will be broadly utilized in industrial enterprises to remotely monitor and control the status of machineries and facilities, and it can also manage the production parameters in industrial processes. However, the current IIoT technologies are still their infancy, and the salient characteristics and requirements for different industries continuously impose many research challenges to address for their industrial use, for example, embedded devices, networking, sensor deployment, cloud computing, big data analysis, security and privacy. In this special section, we solicited original and novel research in all areas of Industrial and Smart Sensor Internet of Things Systems, Applications, and Services.

In a thorough and intensive peer-reviewed process, twenty four manuscripts were selected during first review process. The manuscripts were finally selected for this Special Section after the first and second review processes. Each manuscript selected was blindly reviewed by at least three reviewers consisting of guest editors and external reviewers.

Finally, our thanks go to all editorial staffs and reviewers for their valuable support throughout the preparation and publication of this Special Section. We are most grateful

Cho Jaeik

chojaeik01@gmail.com

1 Security Division, IBM Korea, Seoul, Korea

2 Department of Computer Science and Computer Engineering, La Trobe University, Melbourne, Australia

3 Department of Information Management, Central Police University, Taoyuan 333, Taiwan 
to and would like to thank all of the authors for sharing their insights contributions, and experiences. Additionally, special thanks are due to all the reviewers for their time, effort and valuable criticism and suggestions. 